\title{
Inhibition of Sam68 triggers adipose tissue browning
}

\author{
Junlan Zhou, Min Cheng', Chan Boriboun, Mariam M Ardehali, Changfei Jiang', \\ Qinghua Liu ${ }^{3}$, Shuling Han, David A Goukassian ${ }^{4}$, Yao-Liang Tang ${ }^{5}$, Ting C Zhao ${ }^{6}$, \\ Ming Zhao, Lu Cai ${ }^{7}$, Stéphane Richard ${ }^{8}$, Raj Kishore ${ }^{9}$ and Gangjian Qin
}

Department of Medicine-Cardiology, Feinberg Cardiovascular Research Institute, Northwestern University Feinberg School of Medicine, 303 E Chicago Avenue, Tarry 14-721, Chicago, Illinois 60611, USA

${ }^{1}$ Department of Cardiology, Tongji Medical College, Union Hospital, Huazhong University of Science and Technology, Wuhan, Hubei, China

${ }^{2}$ Department of Biochemistry, University of Ottawa, Ottawa, Ontario, Canada

${ }^{3}$ Institute for Medical Biology and Hubei Provincial Key Laboratory for Protection and Application of Special Plants in Wuling Area of China, College of Life Sciences, South-Central University for Nationalities, Wuhan, Hubei, China ${ }^{4}$ GeneSys Research Institute, CardioVascular Research Center, Tufts University School of Medicine, Boston, Massachusetts, USA

${ }^{5}$ Department of Medicine, Medical College of Georgia, Vascular Biology Center, Georgia Regents University, Augusta, Georgia, USA

${ }^{6}$ Department of Surgery, Roger Williams Medical Center, Boston University Medical School, Providence, Rhode Island, USA

${ }^{7}$ Kosair Children Hospital Research Institute, Departments of Pediatrics, Radiation Oncology,

Pharmacology and Toxicology, University of Louisville, Louisville, Kentucky, USA

${ }^{8}$ Lady Davis Institute for Medical Research, McGill University, Montreal, Quebec, Canada

${ }^{9}$ Center for Translational Medicine, Temple University School of Medicine, Philadelphia, Pennsylvania, USA
Correspondence should be addressed to $G$ Qin

Email

g-qin@northwestern.edu

\begin{abstract}
Obesity is associated with insulin resistance and type 2 diabetes; molecular mechanisms that promote energy expenditure can be utilized for effective therapy. Src-associated in mitosis of $68 \mathrm{kDa}$ (Sam68) is potentially significant, because knockout (KO) of Sam68 leads to markedly reduced adiposity. In the present study, we sought to determine the mechanism by which Sam68 regulates adiposity and energy homeostasis. We first found that Sam68 KO mice have a significantly reduced body weight as compared to controls, and the difference is explained entirely by decreased adiposity. Interestingly, these effects were not mediated by a difference in food intake; rather, they were associated with enhanced physical activity. When they were fed a high-fat diet, Sam68 KO mice gained much less body weight and fat mass than their WT littermates did, and they displayed an improved glucose and insulin tolerance. In Sam68 KO mice, the brown adipose tissue (BAT), inguinal, and epididymal depots were smaller, and their adipocytes were less hypertrophied as compared to their WT littermates. The BAT of Sam68 KO mice exhibited reduced lipid stores and expressed higher levels of Ucp1 and key thermogenic and fatty acid oxidation genes. Similarly, depots of inguinal and epididymal white adipose tissue (WAT) in Sam68 KO mice appeared browner, their multilocular Ucp 1-positive cells were much more abundant, and the expression of Ucp1, Cidea, Prdm16, and Ppargc1a genes was greater as compared to WT controls, which suggests that the loss of Sam68 also promotes WAT browning. Furthermore, in all of the fat
\end{abstract}

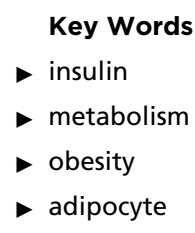

(C) 2015 Society for Endocrinology Printed in Great Britain
Published by Bioscientifica Ltd. 
depots of the Sam68 KO mice, the expression of M2 macrophage markers was up-regulated, and that of M1 markers was down-regulated. Thus, Sam68 plays a crucial role in controlling thermogenesis and may be targeted to combat obesity and associated disorders.

\section{Introduction}

Obesity, which is characterized by abnormal or excessive fat accumulation resulting from energy intake exceeding expenditure, has become a global epidemic and is associated with an array of medical conditions, including insulin resistance, type 2 diabetes, and cardiovascular disease (Bornfeldt \& Tabas 2011). In the body, white adipose tissue (WAT) is a major lipid depot that contains unilocular white adipocytes for storing vast amounts of nutrients as lipids. Brown adipose tissue (BAT), on the other hand, is a key site of energy expenditure via heat production (thermogenesis). The brown adipocytes display multilocular lipid droplet structures and express uncoupling protein 1 (Ucp1), which, when activated, short circuits the electrochemical gradient of ATP production and generates heat (Cypess et al. 2009). Because heat production is an important component of energy expenditure, targeting the molecular and cellular mechanisms that control thermogenesis could be an effective strategy for preventing and treating obesity.

Recently, important advancements have been made in the identification of clusters of $U c p 1$-expressing adipocytes with thermogenic capacity in WAT (Vitali et al. 2012, Wu et al. 2012). These cells, which are called beige or brite fat cells, are defined by their multilocular lipid droplet morphology, high mitochondrial content, and their expression of a core set of brown fat-specific genes (e.g., Ucp1, Cidea, and Pgc1a). The accumulation of beige adipocytes in WAT is referred to as 'browning', and it includes the induction of $U c p 1$ expression and a gene program that gives rise to uncoupled respiration and heat production. Thus, both brown and beige adipocytes are specialized in energy expenditure through thermogenesis (Saito et al. 2009); and evidence suggests that their activities contribute to weight loss and the improvement of diabetes in animals, and they correlate with leanness in humans (Feldmann et al. 2009, Harms \& Seale 2013). At present, a number of genes that activate brown adipocytes and/or drive the induction of beige cells have been reported (Wang et al. 2013, Cohen et al. 2014); however, many of them have not yet been validated (Nedergaard \&
Cannon 2014). Identifying novel factors that induce the white-to-brown transition will not only contribute to a better understanding of the comprehensive regulation of energy homeostasis but also provide potential molecular targets for intervening in obesity and its metabolic consequences.

In addition to environmental temperature and nutrient intake, inflammation is increasingly being recognized as a significant regulator of energy homeostasis (Rao et al. 2014). In fact, obesity is now considered a state of chronic low-grade systemic inflammation (Solinas 2012). In obese animals, fat tissues have been shown to display a substantial degree of inflammation (Shoelson et al. 2006), secrete inflammatory cytokines (Hotamisligil et al. 1993), and harbor various immune cells (Osborn \& Olefsky 2012). More recently, studies have shown that adipose tissue macrophages derived from lean mice express markers of $\mathrm{M} 2$, or 'alternative activated', macrophages. In contrast, obese states are concomitant with M1, or 'classically activated', macrophages (Lumeng et al. 2007). Interestingly, M2 macrophages in the adipose tissues have been shown to secrete catecholamines to induce thermogenic gene expression in BAT and to induce lipolysis in WAT (Nguyen et al. 2011, Qiu et al. 2014), whereas M1 macrophage polarization is pro-inflammatory (Eguchi et al. 2013).

Src-associated in mitosis of $68 \mathrm{kDa}$ (Sam68), also known as KH domain containing, RNA binding, signal transduction associated 1 (Khdrbs1), is the prototype of the STAR (signal transducer and activator of RNA) family of RNA-binding proteins (Richard 2010, Sette 2010). It is involved in a number of cellular processes, including RNA processing (Bielli et al. 2011, Iijima et al. 2011), growth factor receptor and kinase signaling (Huot et al. 2009, Ramakrishnan \& Baltimore 2011), transcription (Zhou et al. 2009, Fu et al. 2013), cell cycle regulation, and apoptosis (Lukong \& Richard 2003, Bielli et al. 2014). Most strikingly, Sam68-null (Sam68 knockout (KO)) mice exhibit a markedly reduced adiposity (Huot et al. 2012), but the underlying mechanism for this is not completely

Published by Bioscientifica Ltd. 
understood. In the present study, we demonstrated that the loss of Sam68 in mice enhances the adipose thermogenic program and triggers WAT browning; these in turn contribute to their lean phenotype, resistance to the induction of obesity, and improved glucose metabolism.

\section{Materials and methods}

\section{Animals}

Sam68 $8^{+/-}$mice on C57BL background were provided by Dr Stéphane Richard (McGill University, Montreal, QC, Canada; Richard et al. 2005) and were bred to obtain Sam68 ${ }^{-1-}$ (Sam68 KO) mice and WT littermate controls. All breeding, maintenance, and experimental procedures were approved by the Institutional Animal Care and Use Committee of Northwestern University and were conducted at the University's Center for Comparative Medicine. Mice were maintained on a $12 \mathrm{~h}$ light: $12 \mathrm{~h}$ darkness cycle and were allowed to feed and drink water ad libitum. Genotypes were determined by PCR of tail DNA using the primers listed in the Supplementary Table S1, see section on supplementary data given at the end of this article. For all of the experiments in the present report, male mice were used. For diet-induced obesity studies, mice were fed with a high-fat diet (HFD; $42 \mathrm{kcal} \%$ fat with high sucrose, 88137, Harlan Teklad, Harlan Laboratories, Inc. IN, USA) for 12 weeks.

\section{Nuclear magnetic resonance analysis of body compositions}

Body compositions were analyzed with Echo MRI Whole Body Composition Analyzer (Echo Medical Systems, Echo MRI Whole Body Composition Analyzer, Houston, TX, USA) in the Comprehensive Metabolic Core of Northwestern University following standard operation procedures.

\section{Open field test of general activity}

The open field arena $(56 \times 56 \mathrm{~cm})$ was used to assess ambulation and anxiety levels in the Behavioral Phenotyping Core of Northwestern University. Briefly, each mouse is placed in the center of the arena, and its ambulation activity is collected by the LimeLight Software (Wilmette, IL, USA) for $20 \mathrm{~min}$. The software provides the total distance traveled as well as the percentage of time/ distance within different parts of the arena. An anxious mouse will spend more of its time along the perimeter of the arena. A hyperactive mouse will have a large ambulation score.

\section{Glucose and insulin tolerance tests}

For the glucose tolerance test (GTT), mice were fasted overnight. Glucose $(2 \mathrm{~g} / \mathrm{kg})$ was administered intraperitoneally, and then blood glucose levels were measured at $0,15,30,60$, and 120 min with a blood glucose meter (Bayer Contour, Whippany, NJ, USA). For the insulin tolerance test (ITT), mice were fasted for $6 \mathrm{~h}$. Insulin $(0.7 \mathrm{U} / \mathrm{kg})$ was administered intraperitoneally, and blood glucose was measured at $0,15,30,60$, and $120 \mathrm{~min}$.

\section{Histology}

Fat tissues were fixed in 10\% neutral buffered formalin and embedded in paraffin. Five micrometer sections were cut, stained with hematoxylin and eosin (H\&E), and photographed under a light microscope. Immunohistochemical staining was performed with anti-Ucp1 antibodies (Abcam, Inc., Cambridge, MA, USA), as described previously by the Rosen laboratory (Kong et al. 2014).

\section{Western blotting}

Fat tissues were collected and immediately frozen in liquid nitrogen. Proteins were extracted with RIPA buffer (Cell Signaling Technology, Inc., Boston, MA, USA) supplemented with complete protease inhibitor cocktail (Roche). For western blotting analyses, protein samples were subjected to SDS-PAGE under reducing conditions, transferred, and blotted with antibodies to IRS1 (Millipore, Temecula, CA, USA), phospho-IRS1 (Tyr612) (Abcam, Inc.), AKT, and phospho-AKT (Ser473) (Cell Signaling Technology, Inc.), and HSP90 (Santa Cruz Biotechnology, Inc.).

\section{Quantitative real-time RT-PCR}

RNA extraction and quantitative real-time RT-PCR (qRT-PCR) were performed as described previously (Tang et al. 2009). Primer sequences are listed in Supplementary Table S1.

\section{Statistical analysis}

All values are reported as means \pm s.E.M. Unpaired twotailed Student's $t$-tests were used for comparisons between two groups. Two-way ANOVAs with repeated measures were used for comparing two factors at multiple levels in the time course studies. A $P$ value of $<0.05$ was considered statistically significant.

Published by Bioscientifica Ltd. 


\section{Results}

\section{Loss of Sam68 protects against diet-induced obesity}

We initiated the present investigation by measuring body weight and composition in Sam68 KO mice and in their WT littermates. Sam68 KO mice displayed significantly reduced body weight and fat mass, and the difference was explained entirely by decreased adiposity, seeing as their lean mass was normal (Fig. 1A and B). Further analyses showed that BAT, inguinal, and epididymal depots were smaller in the Sam68 KO mice (Fig. 1C), and their adipocytes showed less hypertrophy than those of control mice did, and there were no weight changes in the liver or spleen (Supplementary Figure S1, see section on supplementary data given at the end of this article). The effect on adiposity was not mediated by food intake, which did not differ between the two genotypes (Fig. 1D); rather, it was associated with enhanced physical activity (Fig. 1E). Taken together, these data suggest that the Sam68 KO mice were probably leaner because of a significant increase in physical activity that was not related to feeding behavior. Next, we chose Sam68 KO mice and WT controls that were matched for body weights and fed them with an HFD. Notably, the increases in body weight and fat mass were significantly attenuated in Sam68 KO mice as compared to the WT controls (Fig. 2A and $\mathrm{B})$.

\section{Loss of Sam68 results in improved insulin sensitivity}

Diet-induced obesity is frequently associated with glucose imbalance and progressive metabolic dysfunction. Notably, Sam68 KO mice on HFD showed improved glucose and insulin tolerance to a degree that was consistent with their suppressed body weight (Fig. 2C and D). In addition, we assessed the effect of Sam68 on insulin signaling and found that the insulin-induced phosphorylation of IRS1 (Tyr612) and AKT (Ser473) was augmented in the WAT of Sam $68^{-1-}$ mice (Fig. 2E). These findings indicate that the Sam68 KO animals were protected against HFD-induced glucose intolerance and insulin resistance.

\section{Sam68 is required for normal thermogenesis in BAT}

Adipose tissue is an important organ that contributes to glucose homeostasis. BAT has been confirmed recently to play a crucial a role in glucose metabolism (Stanford et al. 2013); thus, we evaluated whether Sam68 expression is required for BAT structure and function. The Sam68 KO
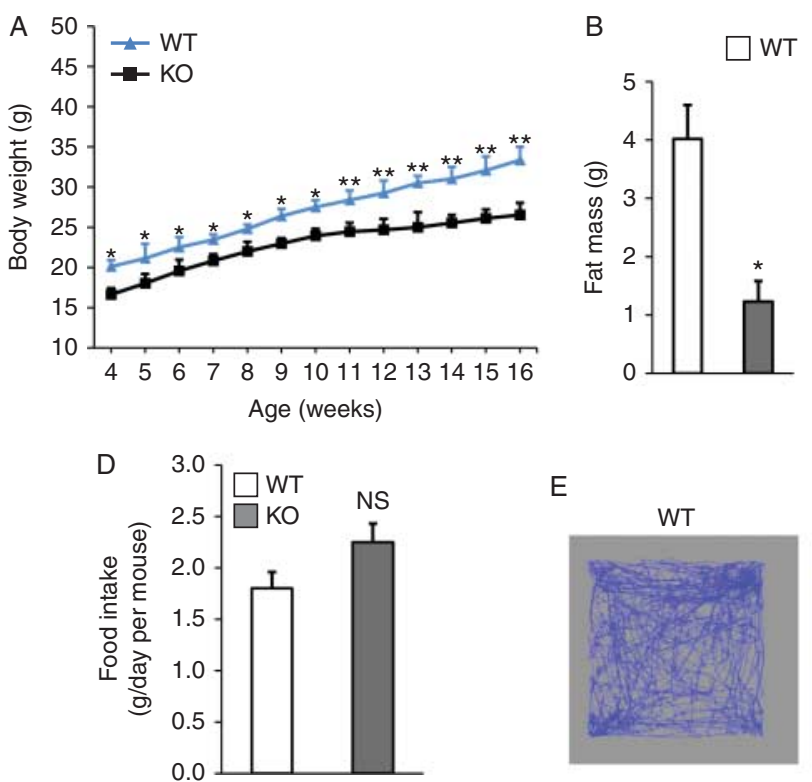

E

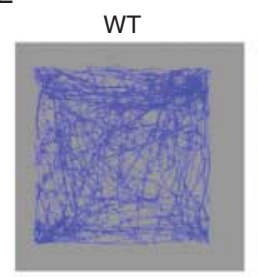

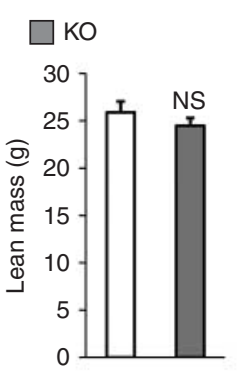
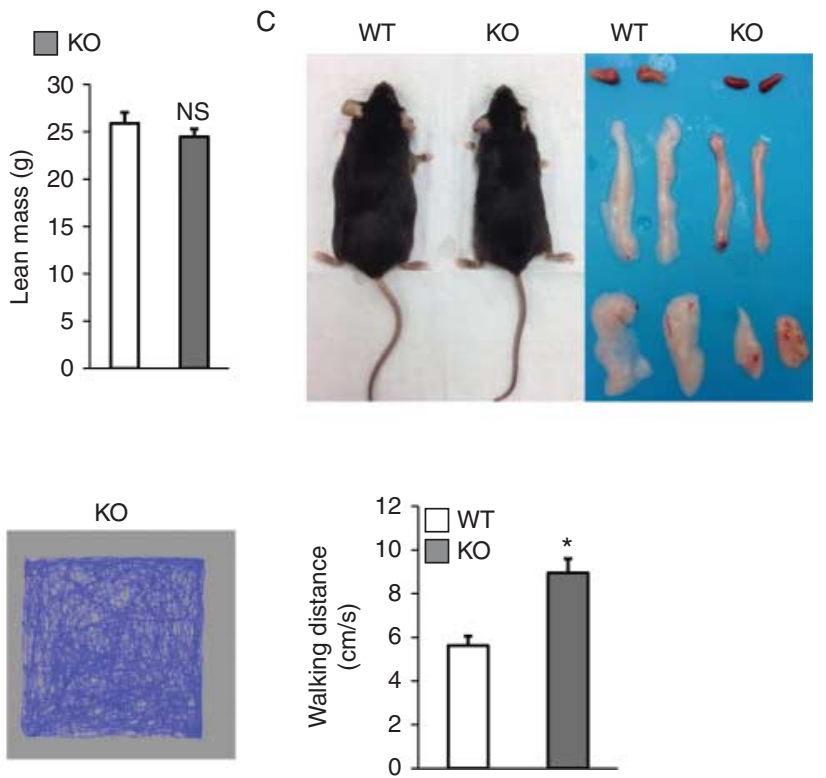

Figure 1

Loss of Sam68 decreases adiposity in mice on regular chow. (A) Body weight of WT mice and Sam68 KO mice ( $n=6-10 /$ group, $* P<0.05$ and $* * P<0.01$ ). (B) Body composition, fat mass (left panel), and lean mass (right panel) as assessed by nuclear magnetic resonance (NMR) in 6-month-old mice ( $n=6-10 /$ group, $* P<0.05$ ). (C) Gross morphology of 6-month-old WT and
(C) 2015 Society for Endocrinology Printed in Great Britain
Sam68 KO mice and BAT, inguinal, and epididymal fat pads. (D) Food intake measured daily for 3 days in 4-month-old mice and averaged ( $n=6 /$ group; NS, not significant). (E) Representative (left and middle panels) and quantification (right panel) of general activity of 6-month-old WT and Sam68 KO mice by open field test $(n=4 /$ group, $* P<0.05)$. 
A

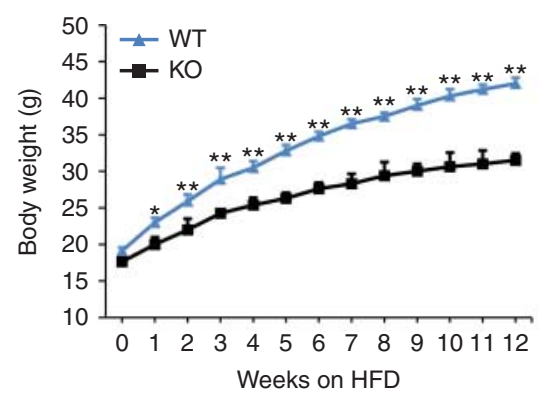

B $\square$ WT $\square$ KO

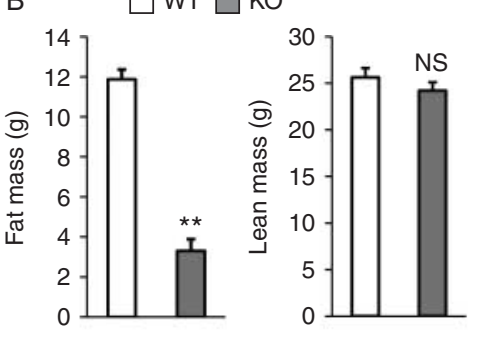

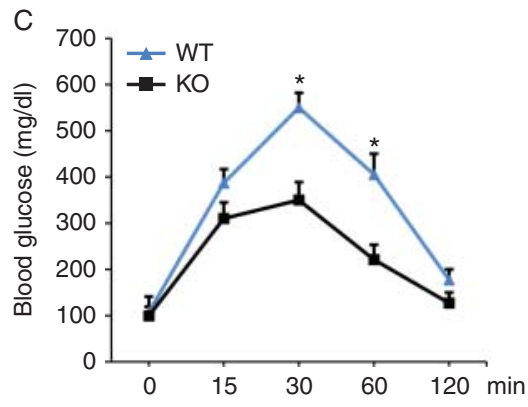

D

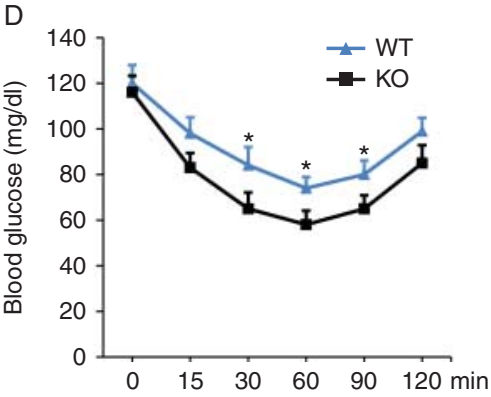

E
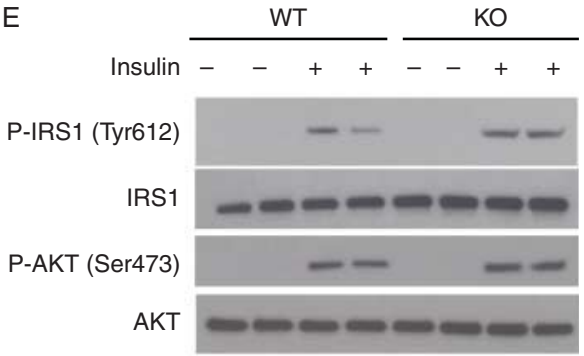

Figure 2

Loss of Sam68 attenuates diet-induced obesity (DIO) and improves insulin sensitivity. Male 8-week-old WT and Sam68 KO mice were first matched with body weight and then fed an HFD for 12 weeks, at which point body composition, GTT, ITT, and insulin signaling in adipose tissues were assessed. (A) Serial measurements of body weight ( $n=6 /$ group, ${ }^{*} P 0.05$

mice had smaller brown adipose depots (Fig. 1C) and reduced lipid stores of BAT at room temperature (Fig. 3A). BAT from Sam68 KO mice maintained at $24^{\circ} \mathrm{C}$ expressed higher levels of Ucp1 than did BAT from control mice (Fig. 3B and Supplementary Figure S2, see section on supplementary data given at the end of this article). This phenotypic change was accompanied by an increased expression of key thermogenic (e.g., Ucp1, Prdm16, Dio2, Cidea, and Elovl3) as well as fatty acid oxidation (e.g., Cidec and $C p t 1 b$ ) genes, but it did not result in a concomitant increase in mitochondrial (e.g., $\beta 3 a r$ and Cox $8 b$ ) or adipose tissue marker (e.g., Fabp4 and Pparg) genes (Fig. 3C). Thus, genetic inactivation of Sam68 leads to enhanced BAT structure, augmented Ucp1 activation, and elevated thermogenic gene expression.

\section{Ablation of Sam68 mice enhances the browning signature of WAT}

Interestingly, depots of inguinal and epididymal WAT of Sam68 KO mice appeared browner than their WT counterparts did (Figs 1C and 4A). Additionally, the weight of various fat depots was lighter in the $\mathrm{KO}$ mice and $* * P<0.01$ ). (B) Body composition by NMR (left panel, fat mass and right panel, lean mass; $n=6 /$ group, $* * P<0.01$ and NS, not significant). (C) GTT and (D) ITT $(n=6, * P<0.05)$. (E) Western blotting analyses of WAT fat depots from WT and Sam68 KO mice 30 min after i.p. injection of insulin.

as compared to control littermates (data not shown). Furthermore, multilocular Ucp1-positive cells were much more abundant, and UCP1 total protein levels were higher in the iWAT and eWAT of Sam68 KO mice than they were in WT control mice (Fig. 4B and Supplementary Figure S2). Consistently, the expression of Ucp1, Cidea, Prdm16, and Ppargc1a was increased in the WAT of the Sam68 KO mice (Fig. 4C and D). Taken together, these results suggest that the deletion of Sam68 promotes adipose browning.

\section{Sam68 KO mice favor M2 macrophage anti-inflammatory phenotypes}

Obesity initiates a state of low-grade inflammation and fibrosis that ultimately predisposes to the progression to insulin resistance and type 2 diabetes (Shoelson et al. 2006, Sun et al. 2011, Osborn \& Olefsky 2012). In particular, a high degree of pro-inflammatory macrophages infiltrates into the adipose tissue during obesity. Thus, we looked at the expression of macrophage markers in different fat depots. As was expected, the mRNA levels of M2 markers (e.g., Arg1 and Il10) were up-regulated, whereas those of M1 markers (e.g., Ccr2, Il1 $\beta$, and Il6) were decreased in all

Published by Bioscientifica Ltd. 
A

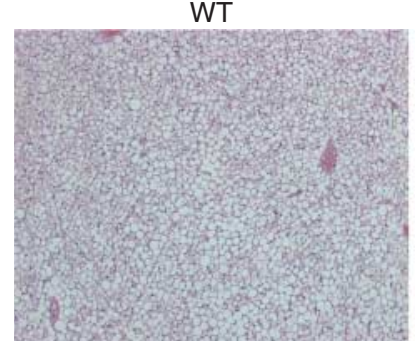

$\mathrm{KO}$

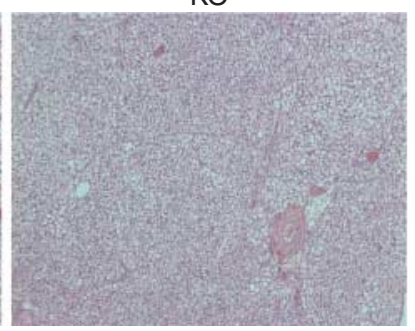

B

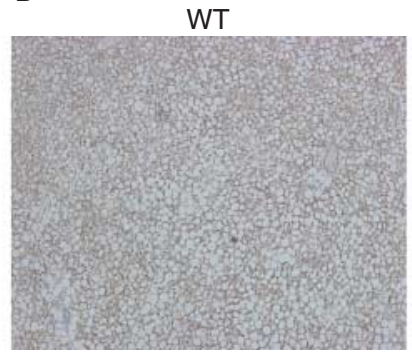

$\mathrm{KO}$

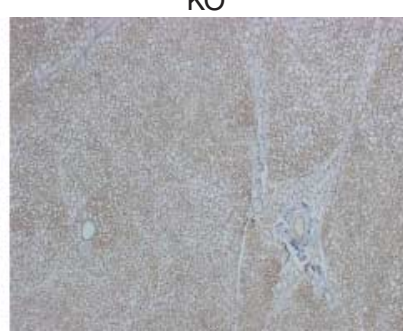

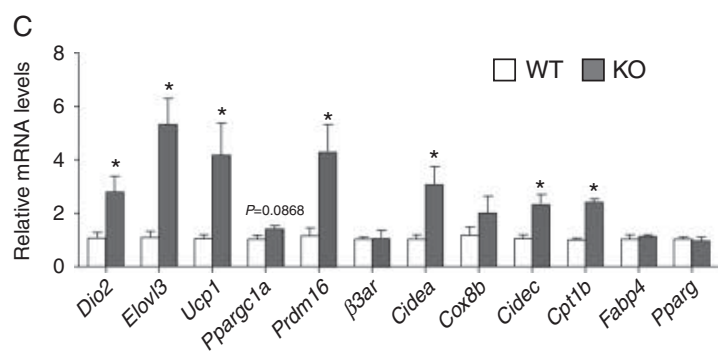

\section{Figure 3}

Sam68 is required for normal thermogenesis in BAT. BAT was isolated from male 10-month-old WT and Sam68 KO mice. (A) H\&E staining of paraffin-embedded sections. Original magnification: $100 \times$.

(B) Immunohistochemical detection of Ucp1 (brown). Original

fat depots (Fig. 5). These data suggest that the loss of Sam68 also leads to macrophages switching so that they augment thermogenic gene expression in BAT and promote browning in WAT.

\section{Discussion}

In the present study, we showed for the first time that the ablation of Sam68 in mice leads to an increase in adipose thermogenic gene expression and WAT browning, which reveals a critical role for Sam68 in the control of thermogenesis and energy expenditure. We further demonstrated that Sam68 KO mice were resistant to dietinduced obesity and display improved insulin sensitivity. Thus, Sam68 provides a novel link between energy homeostasis and obesity.

Sam68 is the prototype of the STAR family of RNAbinding proteins. It has been shown to modulate various cellular signaling and to link that signaling to RNA processing and gene expression (Vogel \& Richard 2012). Sam68 is expressed abundantly in most tissues (Zhou J \& Qin G, unpublished observations), and its structural characteristics permit multiple types of post-translational modifications; this is compatible with its involvement in many cellular functions in different tissue types (Lukong \& Richard 2003, Zhou et al. 2009, Iijima et al. 2011, magnification: $100 \times$. (C) QRT-PCR quantification of mRNA expression. Data are normalized to the mRNA level of ribosomal protein $36 \mathrm{~b} 4$ ( $n=5$ /group, $* P<0.05$ ).

Ramakrishnan \& Baltimore 2011) and in the diverse phenotypes of Sam68-deficient mice (Vogel \& Richard 2012). Sam68-null mice appear to be grossly normal and to have a normal life span; however, they display a higher rate of perinatal death, male infertility, female-delayed mammary gland development, attenuated age-related osteoporosis, and reduced capacity for motor coordination (Vogel \& Richard 2012). Most strikingly, these mice demonstrate reduced adiposity and increased energy expenditure, which have been associated with decreased adipose differentiation (Huot et al. 2012); however, the underlying mechanism for this remains incompletely understood. In the present study, we characterized the fat tissues in Sam68 KO mice and revealed a previously unrecognized function of Sam68 that suppresses browning and thermogenesis. Recently, much has been learned about the types of adipocytes and the core gene program of thermogenesis (van der Lans et al. 2013). The present data identify $\operatorname{Sam} 68$ as a crucial upstream mechanism in the process, and they are also consistent with the notion that energy expenditure through thermogenesis plays a significant role in body weight control.

The present results suggest that $\operatorname{Sam68}$ suppresses thermogenesis, at least in part, by controlling physical activity and by repressing M2 anti-inflammatory cytokine expression. Although physical activity is known to be a robust means of increasing thermogenesis and energy

Published by Bioscientifica Ltd. 
A

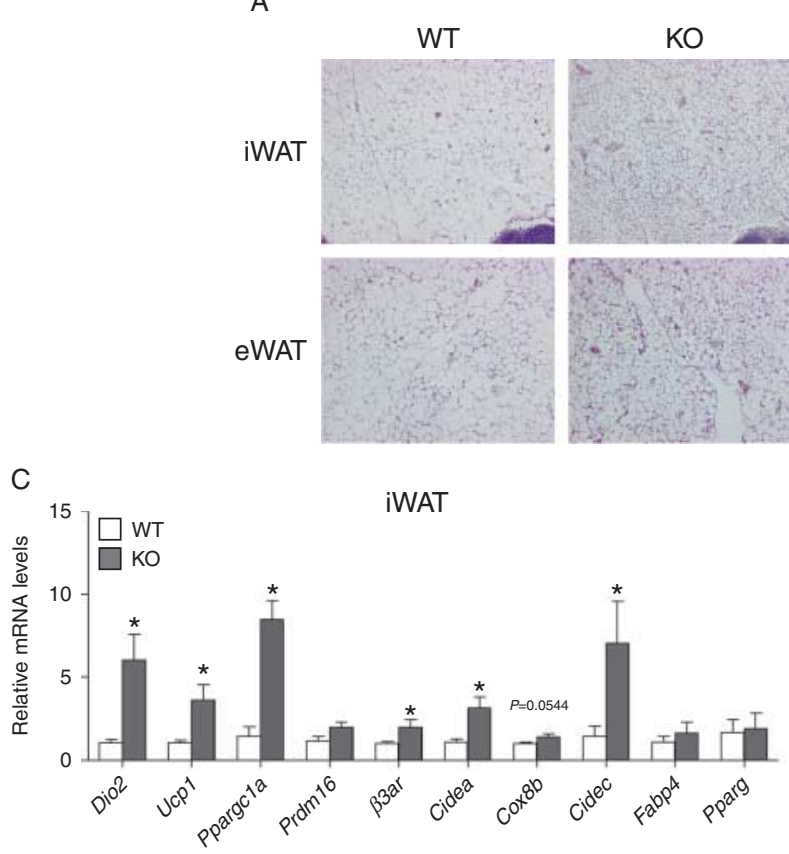

Figure 4

Ablation of Sam68 enhances the browning signature of WAT. Inguinal and epididymal WATs were isolated from male 10-month-old WT and Sam68 KO mice. ( $A$ and $B$ ) H\&E staining (A) and immunohistochemical detection of Ucp1 (brown) (B) in paraffin-embedded sections. Original magnification:

expenditure, the important role of anti-inflammatory mediators has been recognized only recently. For example, the M2 macrophages in the adipose tissues have been shown to participate in thermogenesis via the secretion of catecholamines (Nguyen et al. 2011, Qiu et al. 2014). We found a significantly up-regulated M2 macrophage signature as well as a down-regulated M1 macrophage signature in the adipose tissues of Sam68 KO mice. Interestingly, Sam68
B
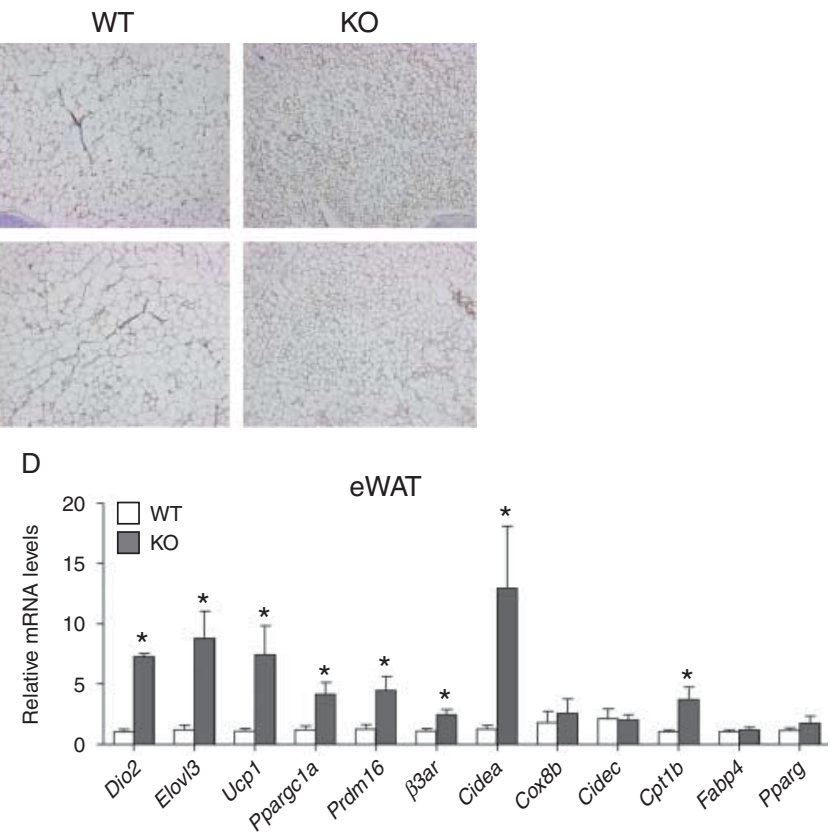

$100 \times$. (C and D) QRT-PCR quantification of mRNA expression in inguinal WAT (C) and epididymal WAT (D). Data are normalized to the $36 \mathrm{~b} 4 \mathrm{mRNA}$ ( $n=5$ /group, $* P<0.05$ ).

has recently been shown to promote NF- $\kappa \mathrm{B}$ activation by $\mathrm{TNF} \alpha$ and to modulate the NF- $\kappa \mathrm{B}$ selection of target promoters in mouse embryonic fibroblasts and activated $\mathrm{T}$ cells respectively (Ramakrishnan \& Baltimore 2011, Fu et al. 2013). Because NF- $\mathrm{BB}$ is an important regulator of macrophage polarization (Porta et al. 2009), it is conceivable that $\mathrm{NF}-\mathrm{\kappa B}$ might be involved in the alteration of the M2-M1 balance in Sam68 KO mice. Taken together, the present data
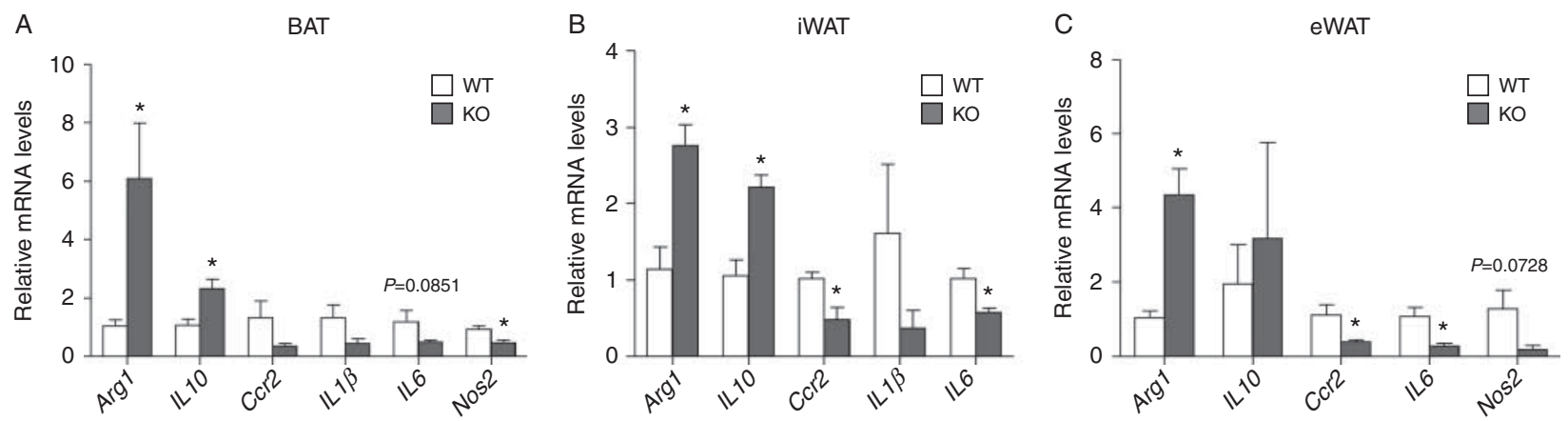

Figure 5

Sam68 KO mice favor M2 macrophage anti-inflammatory phenotypes. RNAs were isolated from the adipose tissues of male 10-month-old WT and Sam68 KO mice. QRT-PCR was performed, and the values were normalized to $36 \mathrm{~b} 4(n=5, * P<0.05)$ for assessment of the inflammatory gene expression in BAT (A), inguinal WAT (B), and epididymal WAT (C). http://joe.endocrinology-journals.org DOI: $10.1530 / \mathrm{JOE}-14-0727$
Published by Bioscientifica Ltd. 
suggest that Sam68 acts to coordinate the activities of multiple tissues to suppress heat generation. Furthermore, the role of Sam68 in the gene expression program may be cell type- and biological context-dependent. Whereas the levels of the adipose genes Ppary and Fabp4 are unaltered in the adipose tissues of Sam68 KO mice, their expressions are down-regulated in 3T3-L1 cells after the knockdown of Sam68 and culture in adipose differentiation conditions (Huot et al. 2012). Ongoing studies are currently under way to dissect the relative contributions from brown fat cells, beige cells, and macrophages as well as the molecular underpinnings of how Sam68 regulates mRNA expression in these different cell types.

In summary, the present study demonstrates that Sam68 plays a crucial role in adipose thermogenesis, WAT browning, and body weight control. Thus, targeting Sam68 in relevant tissues may provide a potential strategy for combating obesity and associated metabolic disorders.

\section{Supplementary data}

This is linked to the online version of the paper at http://dx.doi.org/10.1530/ JOE-14-0727.

\section{Declaration of interest}

The authors declare that there is no conflict of interest that could be perceived as prejudicing the impartiality of the research reported.

\section{Funding}

This work was supported by the National Institute of Health (R01 grant numbers HL093439 and HL113541 to G Q; R01 grant numbers HL053354, HL126186, and HL091883 to R K); the American Heart Association (grant number 13SDG17120011 to J Z); and the Natural Science Foundation of China (grant number 81100084 to M C). This work was also supported by the Northwestern University Mouse Histology and Phenotyping Laboratory and the Cancer Center (support grant number $\mathrm{NCl}$ CA060553) the Behavioral Phenotyping Core.

\section{References}

Bielli P, Busa R, Paronetto MP \& Sette C 2011 The RNA-binding protein Sam68 is a multifunctional player in human cancer. Endocrine-Related Cancer 18 R91-R102. (doi:10.1530/ERC-11-0041)

Bielli P, Busa R, Di Stasi SM, Munoz MJ, Botti F, Kornblihtt AR \& Sette C 2014 The transcription factor FBI-1 inhibits SAM68-mediated BCL-X alternative splicing and apoptosis. EMBO Reports 15 419-427. (doi:10.1002/embr.201338241)

Bornfeldt KE \& Tabas I 2011 Insulin resistance, hyperglycemia, and atherosclerosis. Cell Metabolism 14 575-585. (doi:10.1016/j.cmet.2011.07.015)

Cohen P, Levy Julia D, Zhang Y, Frontini A, Kolodin Dmitriy P, Svensson Katrin J, Lo James C, Zeng X, Ye L, Khandekar Melin J et al. 2014 Ablation of PRDM16 and beige adipose causes metabolic dysfunction and a subcutaneous to visceral fat switch. Cell 156 304-316. (doi:10.1016/j.cell.2013.12.021)
Cypess AM, Lehman S, Williams G, Tal I, Rodman D, Goldfine AB, Kuo FC, Palmer EL, Tseng Y-H, Doria A et al. 2009 Identification and importance of brown adipose tissue in adult humans. New England Journal of Medicine 360 1509-1517. (doi:10.1056/NEJMoa0810780)

Eguchi J, Kong X, Tenta M, Wang X, Kang S \& Rosen ED 2013 Interferon regulatory factor 4 regulates obesity-induced inflammation through regulation of adipose tissue macrophage polarization. Diabetes $\mathbf{6 2}$ 3394-3403. (doi:10.2337/db12-1327)

Feldmann HM, Golozoubova V, Cannon B \& Nedergaard J 2009 UCP1 ablation induces obesity and abolishes diet-induced thermogenesis in mice exempt from thermal stress by living at thermoneutrality. Cell Metabolism 9 203-209. (doi:10.1016/j.cmet.2008.12.014)

Fu K, Sun X, Zheng W, Wier EM, Hodgson A, Tran DQ, Richard S \& Wan F 2013 Sam 68 modulates the promoter specificity of NF- $\kappa$ B and mediates expression of CD25 in activated T cells. Nature Communications 41909. (doi:10.1038/ncomms2916)

Harms M \& Seale P 2013 Brown and beige fat: development, function and therapeutic potential. Nature Medicine 19 1252-1263. (doi:10.1038/ $\mathrm{nm} .3361)$

Hotamisligil GS, Shargill NS \& Spiegelman BM 1993 Adipose expression of tumor necrosis factor- $\alpha$ : direct role in obesity-linked insulin resistance. Science 259 87-91. (doi:10.1126/science.7678183)

Huot ME, Brown CM, Lamarche-Vane N \& Richard S 2009 An adaptor role for cytoplasmic Sam68 in modulating Src activity during cell polarization. Molecular and Cellular Biology 29 1933-1943. (doi:10.1128/ MCB.01707-08)

Huot MÉ, Vogel G, Zabarauskas A, Ngo CT, Coulombe-Huntington J, Majewski J \& Richard S 2012 The Sam68 STAR RNA-binding protein regulates mTOR alternative splicing during adipogenesis. Molecular Cell 46 187-199. (doi:10.1016/j.molcel.2012.02.007)

Iijima T, Wu K, Witte H, Hanno-Iijima Y, Glatter T, Richard S \& Scheiffele P 2011 SAM68 regulates neuronal activity-dependent alternative splicing of neurexin-1. Cell 147 1601-1614. (doi:10.1016/j.cell.2011.11.028)

Kong X, Banks A, Liu T, Kazak L, Rao RR, Cohen P, Wang X, Yu S, Lo JC, Tseng YH et al. 2014 IRF4 is a key thermogenic transcriptional partner of PGC-1 $\alpha$. Cell 158 69-83. (doi:10.1016/j.cell.2014.04.049)

van der Lans AA, Hoeks J, Brans B, Vijgen GH, Visser MG, Vosselman MJ, Hansen J, Jörgensen JA, Wu J, Mottaghy FM et al. 2013 Cold acclimation recruits human brown fat and increases nonshivering thermogenesis. Journal of Clinical Investigation 123 3395-3403. (doi:10.1172/JCI68993)

Lukong KE \& Richard S 2003 Sam68, the KH domain-containing superSTAR. Biochimica et Biophysica Acta 1653 73-86.

Lumeng CN, Bodzin JL \& Saltiel AR 2007 Obesity induces a phenotypic switch in adipose tissue macrophage polarization. Journal of Clinical Investigation 117 175-184. (doi:10.1172/JCI29881)

Nedergaard J \& Cannon B 2014 The browning of white adipose tissue: some burning issues. Cell Metabolism 20 396-407. (doi:10.1016/j.cmet.2014. 07.005)

Nguyen KD, Qiu Y, Cui X, Goh YP, Mwangi J, David T, Mukundan L, Brombacher F, Locksley RM \& Chawla A 2011 Alternatively activated macrophages produce catecholamines to sustain adaptive thermogenesis. Nature 480 104-108. (doi:10.1038/nature10653)

Osborn O \& Olefsky JM 2012 The cellular and signaling networks linking the immune system and metabolism in disease. Nature Medicine $\mathbf{1 8}$ 363-374. (doi:10.1038/nm.2627)

Porta C, Rimoldi M, Raes G, Brys L, Ghezzi P, Di Liberto D, Dieli F, Ghisletti S, Natoli G, De Baetselier P et al. 2009 Tolerance and M2 (alternative) macrophage polarization are related processes orchestrated by p50 nuclear factor $\kappa$ B. PNAS 106 14978-14983. (doi:10.1073/ pnas.0809784106)

Qiu Y, Nguyen Khoa D, Odegaard Justin I, Cui X, Tian X, Locksley Richard M, Palmiter Richard D \& Chawla A 2014 Eosinophils and type 2 cytokine signaling in macrophages orchestrate development of functional beige fat. Cell 157 1292-1308. (doi:10.1016/j.cell.2014. 03.066) 
Ramakrishnan P \& Baltimore D 2011 Sam68 is required for both NF- $\kappa$ B activation and apoptosis signaling by the TNF receptor. Molecular Cell 43 167-179. (doi:10.1016/j.molcel.2011.05.007)

Rao RR, Long JZ, White JP, Svensson KJ, Lou J, Lokurkar I, Jedrychowski MP, Ruas JL, Wrann CD, Lo JC et al. 2014 Meteorin-like is a hormone that regulates immune-adipose interactions to increase beige fat thermogenesis. Cell 157 1279-1291. (doi:10.1016/j.cell.2014.03.065)

Richard S 2010 Reaching for the stars: linking RNA binding proteins to diseases. Advances in Experimental Medicine and Biology 693 142-157.

Richard S, Torabi N, Franco GV, Tremblay GA, Chen T, Vogel G, Morel M, Cléroux P, Forget-Richard A, Komarova S et al. 2005 Ablation of the Sam68 RNA binding protein protects mice from age-related bone loss. PLoS Genetics 1 74-87. (doi:10.1371/journal.pgen.0010074)

Saito M, Okamatsu-Ogura Y, Matsushita M, Watanabe K, Yoneshiro T, Nio-Kobayashi J, Iwanaga T, Miyagawa M, Kameya T, Nakada K et al. 2009 High incidence of metabolically active brown adipose tissue in healthy adult humans: effects of cold exposure and adiposity. Diabetes 58 1526-1531. (doi:10.2337/db09-0530)

Sette C 2010 Post-translational regulation of star proteins and effects on their biological functions. Advances in Experimental Medicine and Biology 693 54-66.

Shoelson SE, Lee J \& Goldfine AB 2006 Inflammation and insulin resistance. Journal of Clinical Investigation 116 1793-1801. (doi:10.1172/ JCI29069)

Solinas G 2012 Molecular pathways linking metabolic inflammation and thermogenesis. Obesity Reviews 13(Suppl 2) 69-82. (doi:10.1111/j.1467789X.2012.01047.x)

Stanford KI, Middelbeek RJ, Townsend KL, An D, Nygaard EB, Hitchcox KM, Markan KR, Nakano K, Hirshman MF, Tseng YH et al. 2013 Brown adipose tissue regulates glucose homeostasis and insulin sensitivity. Journal of Clinical Investigation 123 215-223. (doi:10.1172/JCI62308)

Sun K, Kusminski CM \& Scherer PE 2011 Adipose tissue remodeling and obesity. Journal of Clinical Investigation 121 2094-2101. (doi:10.1172/ JCI45887)

Tang YL, Zhu W, Cheng M, Chen L, Zhang J, Sun T, Kishore R, Phillips MI, Losordo DW \& Qin G 2009 Hypoxic preconditioning enhances the benefit of cardiac progenitor cell therapy for treatment of myocardial infarction by inducing CXCR4 expression. Circulation Research 104 1209-1216. (doi:10.1161/CIRCRESAHA.109.197723)

Vitali A, Murano I, Zingaretti MC, Frontini A, Ricquier D \& Cinti S 2012 The adipose organ of obesity-prone C57BL/6J mice is composed of mixed white and brown adipocytes. Journal of Lipid Research $\mathbf{5 3}$ 619-629. (doi:10.1194/jlr.M018846)

Vogel G \& Richard S 2012 Emerging roles for Sam68 in adipogenesis and neuronal development. RNA Biology 9 1129-1133. (doi:10.4161/rna. 21409)

Wang J, Liu R, Wang F, Hong J, Li X, Chen M, Ke Y, Zhang X, Ma Q, Wang R et al. 2013 Ablation of LGR4 promotes energy expenditure by driving white-to-brown fat switch. Nature Cell Biology 15 1455-1463. (doi:10.1038/ncb2867)

Wu J, Bostrom P, Sparks LM, Ye L, Choi JH, Giang AH, Khandekar M, Virtanen KA, Nuutila P, Schaart G et al. 2012 Beige adipocytes are a distinct type of thermogenic fat cell in mouse and human. Cell $\mathbf{1 5 0}$ 366-376. (doi:10.1016/j.cell.2012.05.016)

Zhou J, Zhu Y, Cheng M, Dinesh D, Thorne T, Poh KK, Liu D, Botros C, Tang YL, Reisdorph N et al. 2009 Regulation of vascular contractility and blood pressure by the E2F2 transcription factor. Circulation 120 1213-1221. (doi:10.1161/CIRCULATIONAHA.109.859207)

Received in final form 14 April 2015

Accepted 22 April 2015

Accepted Preprint published online 1 May 2015
(C) 2015 Society for Endocrinology Printed in Great Britain 